\title{
A $q$-ANALOGUE OF THE WRONSKIAN AND A SECOND SOLUTION OF THE HAHN-EXTON $q$-BESSEL DIFFERENCE EQUATION
}

\author{
R. F. SWARTTOUW AND H. G. MEIJER
}

(Communicated by Hal L. Smith)

\begin{abstract}
A second solution of the $q$-difference equation of the Hahn-Exton $q$-Bessel function, corresponding to the classical $Y_{\nu}(x)$, is found. We introduce a $q$-extension of the Wronskian to determine that the two solutions form a fundamental set.
\end{abstract}

\section{INTRODUCTION}

In mathematics very much attention is paid to the subject of differential equations. However, the theory of $q$-extensions of differential equations has not yet been developed to a great extent. This can partially be explained by the fact that one is not very familiar with $q$-theory and the fact that basic differential equations do not occur frequently in physics. But the most important fact is probably the close relationship with difference equations instead of differential equations. $q$-differential (or $q$-difference) equations may even properly be regarded as a part of the field of difference equations. Results on difference equations may sometimes be transformed into results on $q$-difference equations and vice versa. However, each subject has his own specific problems, and it would therefore be desirable if a book on basic difference equations were available.

It is by no means our aim to give in this paper such a general theory of $q$ difference equations. We will restrict ourselves to the necessary theory in order to give $q$-extensions of the solutions and their mutual relations of the Bessel differential equation. We will obtain a solution, which is a $q$-extension of the classical $Y_{\nu}(x)$. In order to determine if two solutions form a fundamental system, we will derive a $q$-extension of the Wronskian. To our best knowledge, this $q$-Wronskian has not been stated elsewhere, although it is closely related to Casorati's determinant (see, e.g., [7]). So it seems natural to give some general results on the $q$-Wronskian first, before discussing the Hahn-Exton $q$-Bessel difference equation.

\section{THE $q$-WRONSKIAN}

In this section and everywhere else in this paper we will assume that $q$ satisfies the condition $0<q<1$. All functions are defined on $(0, \infty)$.

Received by the editors June 29, 1992.

1991 Mathematics Subject Classification. Primary 33D15, 33D45.

Key words and phrases. Hahn-Exton $q$-Bessel function, $q$-Wronskian, $q$-difference equation.

(C)1994 American Mathematical Society 
A special place in the theory of $q$-difference equations take the so-called $q$-periodic functions. A $q$-periodic function is the solution of the first-order $q$-difference equation

$$
D_{q} f(x)=0, \quad x>0,
$$

where the $q$-difference operator $D_{q}$ is defined by

$$
D_{q} f(x)=\frac{f(x)-f(q x)}{x(1-q)} .
$$

Solutions of (1) thus have the property that $f(q x)=f(x)$ for all $x>0$. It is obvious that the constant function is a solution of (1). Another example of a $q$-periodic function is

$$
f(x)=\sin (\alpha \log x) \quad \text { with } \alpha \log q=2 \pi,
$$

which has an awful singularity at $x=0$.

In the next section, where we will consider solutions of a $q$-Bessel difference equation, the class of solutions has a special form. We shall now show that the only $q$-periodic functions in that class of functions are the constant functions. Let $\phi$ denote a $q$-periodic function. We consider three cases:

(1) Suppose $\lim _{x \downarrow 0} \phi(x)=L$ exists. Then for all $x>0$ we have

$$
\phi(x)=\phi(q x)=\cdots=\phi\left(q^{n} x\right)=\lim _{n \rightarrow \infty} \phi\left(q^{n} x\right)=L,
$$

so $\phi$ is the constant function.

(2) Suppose $\phi$ has the form

$$
\phi(x)=x^{\mu} \sum_{n=0}^{\infty} a_{n} x^{n}, \quad x>0,
$$

with $a_{0} \neq 0$. Then if $\mu \geq 0$, the limit $\lim _{x \downarrow 0} \phi(x)$ exists, so we are in case (1) and $\phi(x)$ is a constant. If $\mu<0$, we consider the function $h(x)=x^{-\mu} \phi(x)$. We have $\lim _{x \downarrow 0} h(x)=a_{0} \neq 0$. On the other hand, we have $h\left(q^{n} x\right)=$ $x^{-\mu} q^{-n \mu} \phi\left(q^{n} x\right)=q^{-n \mu} x^{-\mu} \phi(x)=q^{-n \mu} h(x)$. Hence, the limit $n \rightarrow \infty$ yields $a_{0}=0 \cdot h(x)=0$, which is a contradiction. So a $q$-periodic function cannot be of the form $x^{\mu} \sum_{n=0}^{\infty} a_{n} x^{n}$, with $a_{0} \neq 0, \mu<0$.

(3) Suppose $\phi$ has the form

$$
\phi(x)=x^{\mu} \log x \sum_{n=0}^{\infty} a_{n} x^{n}, \quad x>0,
$$

with $a_{0} \neq 0$. Then if $\mu>0$, the limit $\lim _{x \downarrow 0} \phi(x)=0$ exists, so we are in case (1) and $\phi(x)$ is a constant. If $\mu \leq 0$, we consider the function $h(x)=$ $x^{-\mu} \phi(x)-a_{0} \log x$. Then $\lim _{x \downarrow 0} h(x)=0$. On the other hand, $h\left(q^{n} x\right)=$ $q^{-n \mu} x^{-\mu} \phi(x)-a_{0} \log x-a_{0} n \log q$. The limit $n \rightarrow \infty$ now shows that $a_{0}=$ 0 , which is a contradiction. So a $q$-periodic function cannot be of the form $x^{\mu} \log x \sum_{n=0}^{\infty} a_{n} x^{n}$, with $\mu \leq 0, a_{0} \neq 0$.

So as long as we work in the class of functions of the form $x^{\mu} \sum_{n=0}^{\infty} a_{n} x^{n}$ or $x^{\mu} \log x \sum_{n=0}^{\infty} a_{n} x^{n}$, the only $q$-periodic functions are constant functions.

Now consider the first-order linear $q$-difference equation

$$
D_{q} y(x)+a(x) y(x)=0, \quad x>0,
$$

where $a(x)$ is analytic on the open interval $(0, \infty)$. 
Theorem 1. Let $y_{0}$ denote a solution of (3) such that $y_{0}(x) \neq 0$ for all $x>0$. Then the complete solution of (3) can be represented by $y(x)=\phi(x) y_{0}(x)$, where $\phi$ is an arbitrary q-periodic function.

Proof. An easy consequence of the definition (2) is the $q$-analogue of the product rule for differentiation

$$
D_{q}[f(x) g(x)]=f(q x) D_{q} g(x)+g(x) D_{q} f(x) .
$$

Let $y$ denote a solution of (3). Put $y(x)=y_{0}(x) \phi(x)$. Then using (4)

$$
\begin{aligned}
0 & =D_{q} y(x)+a(x) y(x) \\
& =y_{0}(q x) D_{q} \phi(x)+\phi(x) D_{q} y_{0}(x)+a(x) \phi(x) y_{0}(x)=y_{0}(q x) D_{q} \phi(x) .
\end{aligned}
$$

Hence, $D_{q} \phi(x)=0$ and $\phi(x)$ is a $q$-periodic function. On the other hand, if $\phi$ is a $q$-periodic function, then $y(x)=\phi(x) y_{0}(x)$ is a solution of (3).

We will now consider the second-order linear $q$-difference equation

$$
D_{q}^{2} y(x)+a(x) D_{q} y(x)+b(x) y(x q)=0, \quad x>0,
$$

where $a(x)$ and $b(x)$ are analytic on the open interval $(0, \infty)$ and $D_{q}^{2}$ is defined by

$$
D_{q}^{2} f(x)=D_{q}\left(D_{q} f(x)\right)=\frac{q f(x)-(1+q) f(q x)+f\left(q^{2} x\right)}{q(1-q)^{2} x^{2}} .
$$

Equation (5) can be rewritten as

$$
y\left(q^{2} x\right)+A(x) y(q x)+B(x) y(x)=0, \quad x>0,
$$

where

$$
\left\{\begin{array}{l}
A(x)=-(1+q)-a(x)(1-q) x q+b(x)(1-q)^{2} x^{2} q \\
B(x)=q+a(x)(1-q) x q
\end{array}\right.
$$

We assume that $A(x)$ and $B(x)$ have no positive zeros.

If $f_{1}$ and $f_{2}$ are two solutions of (5) and if $\phi_{1}$ and $\phi_{2}$ are $q$-periodic functions, then it is obvious that $f(x)=\phi_{1}(x) f_{1}(x)+\phi_{2}(x) f_{2}(x)$ is a solution of (5). Two solutions $f_{1}$ and $f_{2}$ of (5) are called a fundamental system of solutions of (5) if every solution $f$ of (5) can be written as $f=\phi_{1} f_{1}+\phi_{2} f_{2}$, where $\phi_{1}$ and $\phi_{2}$ are $q$-periodic functions.

To determine if two solutions of the linear $q$-difference equation (5) form a fundamental system, we introduce a $q$-analogue of the Wronskian.

Definition. If $f_{1}$ and $f_{2}$ are two solutions of the linear $q$-difference equation (5), we define the $q$-Wronskian by

$$
\begin{aligned}
& W_{q}\left(f_{1}(x), f_{2}(x)\right) \stackrel{\text { def }}{=}\left|\begin{array}{cc}
f_{1}(x) & f_{2}(x) \\
D_{q} f_{1}(x) & D_{q} f_{2}(x)
\end{array}\right| \\
&=f_{1}(x) D_{q} f_{2}(x)-f_{2}(x) D_{q} f_{1}(x) .
\end{aligned}
$$

It is easy to see that, if $q \uparrow 1$, the $q$-Wronskian tends to the ordinary Wronskian

$$
W\left(f_{1}(x), f_{2}(x)\right)=\left|\begin{array}{ll}
f_{1}(x) & f_{2}(x) \\
f_{1}^{\prime}(x) & f_{2}^{\prime}(x)
\end{array}\right|=f_{1}(x) \frac{d f_{2}(x)}{d x}-f_{2}(x) \frac{d f_{1}(x)}{d x} .
$$


Remark 1. When we apply the definition of the $q$-difference operator (2) to the $q$-Wronskian, (8) can be rewritten in the form

$$
W_{q}\left(f_{1}(x), f_{2}(x)\right)=\frac{f_{1}(x q) f_{2}(x)-f_{1}(x) f_{2}(x q)}{x(1-q)} .
$$

Remark 2. By writing out the definition it is easy to see that the $q$-Wronskian has the properties

$$
W_{q}\left(f(x), c_{1} g_{1}(x)+c_{2} g_{2}(x)\right)=c_{1} W_{q}\left(f(x), g_{1}(x)\right)+c_{2} W_{q}\left(f(x), g_{2}(x)\right)
$$

and

$$
W_{q}(f(x), f(x))=0 .
$$

Theorem 2 ( $q$-analogue of Abel's theorem). Let $f_{1}$ and $f_{2}$ be solutions of the $q$-difference equation (5), and let the q-Wronskian be defined by (8). Then it satisfies the linear first-order q-difference equation

$$
D_{q} W_{q}\left(f_{1}(x), f_{2}(x)\right)+a(x) W_{q}\left(f_{1}(x), f_{2}(x)\right)=0 .
$$

Proof. By the $q$-product rule (4) and by (8) we have

$$
\begin{aligned}
D_{q} W_{q} & \left\{f_{1}(x), f_{2}(x)\right\}=D_{q}\left\{f_{1}(x) D_{q} f_{2}(x)-f_{2}(x) D_{q} f_{1}(x)\right\} \\
= & f_{1}(x q) D_{q}^{2} f_{2}(x)+D_{q} f_{2}(x) D_{q} f_{1}(x)-f_{2}(x q) D_{q}^{2} f_{1}(x)-D_{q} f_{1}(x) D_{q} f_{2}(x) \\
= & f_{1}(x q)\left\{-a(x) D_{q} f_{2}(x)-b(x) f_{2}(x q)\right\} \\
& -f_{2}(x q)\left\{-a(x) D_{q} f_{1}(x)-b(x) f_{1}(x q)\right\} \\
= & -a(x)\left\{f_{1}(x q) D_{q} f_{2}(x)-f_{2}(x q) D_{q} f_{1}(x)\right\} \\
= & -a(x)\left\{f_{1}(x) D_{q} f_{2}(x)-f_{2}(x) D_{q} f_{1}(x)\right\}=-a(x) W_{q}\left(f_{1}(x), f_{2}(x)\right) .
\end{aligned}
$$

A theorem concerning the relation between the $q$-Wronskian and a fundamental system is as follows.

Theorem 3. Let $f_{1}$ and $f_{2}$ be solutions of the q-difference equation (5), and let the $q$-Wronskian be defined by (8). Then every solution $f$ of $(5)$ can be written as $f(x)=\phi_{1}(x) f_{1}(x)+\phi_{2}(x) f_{2}(x)$, with $\phi_{1}$ and $\phi_{2}$ q-periodic functions, if and only if the $q$-Wronskian $W_{q}\left(f_{1}(x), f_{2}(x)\right) \neq 0$ for all $x>0$.

Proof. All functions are defined on the open interval $(0, \infty)$. We consider for fixed $x$ the set $G(x)=\left\{q^{n} x \mid n \in \mathbb{Z}\right\}$. On this set $q$-periodic functions are constants. A solution $f$ of $(6)$ and hence of (5) is completely determined by $f(x)$ and $f(q x)$. Now we have: Every solution $f$ can be written as $f=$ $c_{1} f_{1}+c_{2} f_{2}$ (with $c_{1}$ and $c_{2}$ constants) on the set $G$

$$
\begin{aligned}
& \Leftrightarrow\left\{\begin{array}{l}
f(x)=c_{1} f_{1}(x)+c_{2} f_{2}(x) \\
f(x q)=c_{1} f_{1}(x q)+c_{2} f_{2}(x q)
\end{array} \Leftrightarrow\left|\begin{array}{cc}
f_{1}(x) & f_{2}(x) \\
f_{1}(x q) & f_{2}(x q)
\end{array}\right| \neq 0\right. \\
& \Leftrightarrow W_{q}\left(f_{1}(x), f_{2}(x)\right) \neq 0 .
\end{aligned}
$$

\section{The Hahn-Exton $q$-Bessel Difference eQUation}

In the literature several $q$-analogues of the Bessel function

$$
J_{\nu}(x)=\left(\frac{x}{2}\right)^{\nu} \sum_{k=0}^{\infty} \frac{(-1)^{k}(x / 2)^{2 k}}{\Gamma(\nu+k+1) k !}
$$


have been studied. In [1, 2] Exton derived the function

$$
C_{\alpha}(x ; q)=\sum_{n=0}^{\infty} \frac{(-1)^{n} q^{n(n-1) / 2} x^{n}(1-q)^{n}}{\Gamma_{q}(\alpha+n+1)(q ; q)_{n}}
$$

as a solution of a basic analogue of the Bessel-Clifford equation. Here

$$
\left\{\begin{array}{l}
(a ; q)_{0}=1, \\
(a ; q)_{n}=(1-a)(1-a q) \cdots\left(1-a q^{n-1}\right), \quad n=1,2, \ldots, \infty,
\end{array}\right.
$$

and the $q$-Gamma function $\Gamma_{q}(x)$ is defined by

$$
\Gamma_{q}(x)=\frac{(q ; q)_{\infty}}{\left(q^{x} ; q\right)_{\infty}}(1-q)^{1-x}
$$

If we take the limit $q \uparrow 1$ and use the fact that $\lim _{q \uparrow 1} \Gamma_{q}(x)=\Gamma(x)$ (see [3] for more details of this limit), an easy calculation shows that $C_{\alpha}(x ; q)$ is a $q$-extension of $(x / 2)^{-\alpha / 2} J_{\alpha}(2 \sqrt{x})$, where $J_{\alpha}(x)$ is the classical Bessel function defined by (14). Recently Vaksman and Korogodskii [9] gave an interpretation of the $q$-Bessel functions (15) as matrix elements of irreducible representations of the quantum group of plane motions.

Nowadays it is common to consider a slightly modified form of (15), first investigated by Koornwinder and Swarttouw (see [6, 8]) and also considered by Koelink [5]. This Hahn-Exton q-Bessel function (the name of W. Hahn is involved since he investigated the special case $\alpha=0$ of (15) in [4]) is defined by

$$
J_{\nu}(x ; q)=x^{\nu} \frac{\left(q^{\nu+1} ; q\right)_{\infty}}{(q ; q)_{\infty}} \sum_{n=0}^{\infty} \frac{(-1)^{n} q^{n(n+1) / 2} x^{2 n}}{\left(q^{\nu+1} ; q\right)_{n}(q ; q)_{n}} .
$$

If in (16) $x$ is replaced by $x(1-q) / 2$ and if $q \uparrow 1$, we find the classical Bessel function (14). In this paper we consider the $q$-Bessel function defined by (16).

It is easy to check that $J_{\nu}(x ; q)$ satisfies the relation

$$
y(x q)+q^{-\nu / 2}\left(q x^{2}-1-q^{\nu}\right) y\left(x q^{1 / 2}\right)+y(x)=0 .
$$

Using (7), relation (17) can be rewritten as a $q$-difference equation

$$
\begin{aligned}
& q^{1 / 2} x^{2}\left(1-q^{1 / 2}\right)^{2} D_{q^{1 / 2}}^{2} y(x)+x\left(1-q^{1 / 2}\right)^{2} D_{q^{1 / 2}} y(x) \\
& \quad+\left(x^{2} q^{1-\nu / 2}+\left(1-q^{\nu / 2}\right)\left(1-q^{-\nu / 2}\right)\right) y\left(x q^{1 / 2}\right)=0
\end{aligned}
$$

where

$$
D_{q^{1 / 2}} y(x)=\frac{y(x)-y\left(q^{1 / 2} x\right)}{x\left(1-q^{1 / 2}\right)}
$$

Let $f_{1}$ and $f_{2}$ denote two solutions of (18). The $q$-analogue of Abel's theorem (13) implies that for the $q^{1 / 2}$-Wronskian we have the relation

$$
D_{q^{1 / 2}} W_{q^{1 / 2}}\left(f_{1}(x), f_{2}(x)\right)+\frac{1}{x q^{1 / 2}} W_{q^{1 / 2}}\left(f_{1}(x), f_{2}(x)\right)=0 \text {. }
$$

It is easy to verify that $W_{q^{1 / 2}}=1 / x$ is a solution of (19). By Theorem 1 we have

$$
W_{q^{1 / 2}}\left(f_{1}, f_{2}\right)=\frac{\phi\left(f_{1}, f_{2}\right)}{x}
$$

where $\phi$ is a $q^{1 / 2}$-periodic function depending on $f_{1}$ and $f_{2}$. 
If we replace $\nu$ by $-\nu$ and $x$ by $x q^{-\nu / 2}$ in (18), we obtain that $J_{-\nu}\left(x q^{-\nu / 2} ; q\right)$ is a second solution of (18) and of (17). Using (10) we have for the $q^{1 / 2}$-Wronskian of $J_{\nu}(x ; q)$ and $J_{-\nu}\left(x q^{-\nu / 2} ; q\right)$

$$
\begin{aligned}
W_{q^{1 / 2}} & \left(J_{\nu}(x ; q), J_{-\nu}\left(x q^{-\nu / 2} ; q\right)\right) \\
\quad= & \frac{J_{\nu}\left(x q^{1 / 2} ; q\right) J_{-\nu}\left(x q^{-\nu / 2} ; q\right)-J_{\nu}(x ; q) J_{-\nu}\left(x q^{(1-\nu) / 2} ; q\right)}{x\left(1-q^{1 / 2}\right)} \\
= & \frac{\phi}{x\left(1-q^{1 / 2}\right)} .
\end{aligned}
$$

With the series representation (16) we obtain

$$
\begin{aligned}
\phi & =\frac{\left(q^{\nu+1} ; q\right)_{\infty}\left(q^{-\nu+1} ; q\right)_{\infty}}{(q ; q)_{\infty}(q ; q)_{\infty}}\left(q^{\nu(\nu+1) / 2}-q^{\nu(\nu-1) / 2}\right) \\
& =-q^{\nu(\nu-1) / 2} \frac{\left(q^{\nu} ; q\right)_{\infty}\left(q^{-\nu+1} ; q\right)_{\infty}}{(q ; q)_{\infty}(q ; q)_{\infty}} .
\end{aligned}
$$

It follows that

$$
\begin{aligned}
& W_{q^{1 / 2}}\left(J_{\nu}(x ; q), J_{-\nu}\left(x q^{-\nu / 2} ; q\right)\right) \\
& \quad=\frac{-q^{\nu(\nu-1) / 2}\left(q^{\nu} ; q\right)_{\infty}\left(q^{-\nu+1} ; q\right)_{\infty}}{x\left(1-q^{1 / 2}\right)(q ; q)_{\infty}(q ; q)_{\infty}}=\frac{-q^{\nu(\nu-1) / 2}\left(1+q^{1 / 2}\right)}{x \Gamma_{q}(\nu) \Gamma_{q}(1-\nu)} .
\end{aligned}
$$

Note that the $q^{1 / 2}$-Wronskian is never zero if $\nu \notin \mathbb{Z}$. By Theorem 3 this implies that if $\nu \notin \mathbb{Z}$ then every solution $f$ of $(17)$ can be written as $f(x)=$ $\phi_{1}(x) J_{\nu}(x ; q)+\phi_{2}(x) J_{-\nu}\left(x q^{-\nu / 2} ; q\right)$, where $\phi_{1}$ and $\phi_{2}$ are $q^{1 / 2}$-periodic functions. If we restrict ourselves to solutions of the form $x^{\mu} \sum_{n=0}^{\infty} a_{n} x^{n}$, then every solution of (17) can be written as $c_{1} J_{\nu}(x ; q)+c_{2} J_{-\nu}\left(x q^{-\nu / 2} ; q\right)$, where $c_{1}$ and $c_{2}$ are constants. If $\nu=n \in \mathbb{Z}$, however, the $q$-Wronskian is identically zero. In that case an easy calculation shows a clear linear dependence. We have

$$
J_{-n}\left(x q^{-n / 2} ; q\right)=(-1)^{n} q^{n / 2} J_{n}(x ; q) .
$$

These properties match with the $q=1$ case.

If we replace in (20) $x$ by $x(1-q) / 2$ and if we let $q \uparrow 1$ and if we use the well-known relation

$$
\frac{1}{\Gamma(\nu) \Gamma(1-\nu)}=\frac{\sin (\pi \nu)}{\pi},
$$

we obtain the classical Wronskian

$$
W\left(J_{\nu}(x), J_{-\nu}(x)\right)=\frac{-2}{\pi x} \sin (\pi \nu) .
$$

Our next aim is to look for a second solution of the Hahn-Exton $q$-Bessel equation which is a $q$-extension of the classical $Y_{\nu}(x)$, defined for $\nu \notin \mathbb{Z}$ by

$$
Y_{\nu}(x)=\frac{\cos (\pi \nu) J_{\nu}(x)-J_{-\nu}(x)}{\sin (\pi \nu)}
$$

and for $n \in \mathbb{Z}$ by

$$
Y_{n}(x)=\lim _{\nu \rightarrow n} Y_{\nu}(x)
$$


Consider the function $Y_{\nu}(x ; q)$, defined for $\nu \notin \mathbb{Z}$ by

$$
Y_{\nu}(x ; q)=\frac{\Gamma_{q}(\nu) \Gamma_{q}(1-\nu)}{\pi}\left\{\cos (\pi \nu) q^{\nu / 2} J_{\nu}(x ; q)-J_{-\nu}\left(x q^{-\nu / 2} ; q\right)\right\} .
$$

For $n \in \mathbb{Z}$ we define

$$
Y_{n}(x ; q)=\lim _{\nu \rightarrow n} Y_{\nu}(x ; q) .
$$

If $\nu \notin \mathbb{Z}$, it is easy to show with relation (22) and the identity $\Gamma\left(\frac{1}{2}\right)=\sqrt{\pi}$ that

$$
\lim _{q \uparrow 1} Y_{\nu}(x(1-q) / 2 ; q)=Y_{\nu}(x) \text {. }
$$

The limit (24), however, is more complicated, but it exists as we will show below.

Observe that for $\nu \notin \mathbb{Z}$

$$
Y_{-\nu}\left(x q^{-\nu / 2} ; q\right)=-\frac{\Gamma_{q}(-\nu) \Gamma_{q}(1+\nu)}{\pi}\left\{J_{\nu}(x ; q)-\cos (\pi \nu) q^{-\nu / 2} J_{-\nu}\left(x q^{-\nu / 2} ; q\right)\right\} \text {. }
$$

Since

$$
\Gamma_{q}(-\nu) \Gamma_{q}(1+\nu)=-q^{\nu} \Gamma_{q}(\nu) \Gamma_{q}(1-\nu)
$$

we have

$Y_{-\nu}\left(x q^{-\nu / 2} ; q\right)=q^{\nu / 2} \frac{\Gamma_{q}(\nu) \Gamma_{q}(1-\nu)}{\pi}\left\{q^{\nu / 2} J_{\nu}(x ; q)-\cos (\pi \nu) J_{-\nu}\left(x q^{-\nu / 2} ; q\right)\right\}$.

This implies for $n \in \mathbb{Z}$ that

$$
Y_{-n}\left(x q^{-n / 2} ; q\right)=(-1)^{n} q^{n / 2} Y_{n}(x ; q) .
$$

Now we will calculate the limit (24), which in particular implies that it exists. Because of relation (25), it is sufficient to consider $Y_{n}(x ; q)$ for $n \geq 0$. To simplify the notation we introduce the function $F$ defined by

$$
F(\nu)=\left(q^{\nu} ; q\right)_{\infty}
$$

Then for $n=0,1,2, \ldots$

$$
F(-n)=0
$$

and

$$
\begin{aligned}
F^{\prime}(-n) & =\left(1-q^{-n}\right) \cdots\left(1-q^{-1}\right)(-\log q)(q ; q)_{\infty} \\
& =(-1)^{n+1} q^{-n(n+1) / 2}(q ; q)_{n}(q ; q)_{\infty} \log q .
\end{aligned}
$$

Substituting this function $F$ in definition (23) we obtain for $\nu \notin \mathbb{Z}$

$$
\begin{array}{r}
Y_{\nu}(x ; q)=\frac{(q ; q)_{\infty}(1-q)}{\pi F(\nu) F(1-\nu)}\left\{\cos (\pi \nu) q^{\nu / 2} x^{\nu} \sum_{k=0}^{\infty} \frac{(-1)^{k} q^{k(k+1) / 2} x^{2 k} F(\nu+k+1)}{(q ; q)_{k}}\right. \\
\left.-x^{\nu} \sum_{k=0}^{\infty} \frac{(-1)^{k} q^{k(k+1) / 2} x^{2 k} q^{-\nu k+\nu^{2} / 2} F(-\nu+k+1)}{(q ; q)_{k}}\right\} .
\end{array}
$$

Applying l'Hôpital's rule we get for $n=0,1,2, \ldots$

$$
Y_{n}(x ; q)=\frac{(q ; q)_{\infty}(1-q)}{\pi} \frac{N_{n}}{D_{n}} .
$$


Here

$$
\begin{gathered}
D_{n}=\left.\frac{d}{d \nu} F(\nu) F(1-\nu)\right|_{\nu=n}=F^{\prime}(n) F(1-n)-F(n) F^{\prime}(1-n) \\
\stackrel{(27)}{=} \begin{cases}F^{\prime}(0) F(1) & \text { if } n=0, \\
-F(n) F^{\prime}(1-n) & \text { if } n \geq 1 .\end{cases}
\end{gathered}
$$

Applying (26) and (28) we have for $n \geq 1$

$$
\begin{aligned}
-F(n) F^{\prime}(1-n) & =-\left(q^{n} ; q\right)_{\infty}(-1)^{n} q^{-n(n-1) / 2}(q ; q)_{n-1}(q ; q)_{\infty} \log q \\
& =(-1)^{n+1} q^{-n(n-1) / 2}(q ; q)_{\infty}^{2} \log q .
\end{aligned}
$$

Moreover,

$$
F^{\prime}(0) F(1)=-(q ; q)_{\infty}(q ; q)_{\infty} \log q .
$$

Hence for $n=0,1,2, \ldots$

$$
D_{n}=(-1)^{n+1} q^{-n(n-1) / 2}(q ; q)_{\infty}^{2} \log q .
$$

On the other hand,

$$
\begin{aligned}
N_{n}= & \frac{1}{2}(-1)^{n} q^{n / 2} x^{n} \log q \sum_{k=0}^{\infty} \frac{(-1)^{k} q^{k(k+1) / 2} x^{2 k} F(n+k+1)}{(q ; q)_{k}} \\
& +q^{n / 2}(-1)^{n} x^{n} \log x \sum_{k=0}^{\infty} \frac{(-1)^{k} q^{k(k+1) / 2} x^{2 k} F(n+k+1)}{(q ; q)_{k}} \\
& +q^{n / 2}(-1)^{n} x^{n} \sum_{k=0}^{\infty} \frac{(-1)^{k} q^{k(k+1) / 2} x^{2 k} F^{\prime}(n+k+1)}{(q ; q)_{k}} \\
& +x^{-n} \log x \sum_{k=0}^{\infty} \frac{(-1)^{k} q^{k(k+1) / 2} x^{2 k} F(-n+k+1) q^{-n k+n^{2} / 2}}{(q ; q)_{k}} \\
& +x^{-n} \sum_{k=0}^{\infty} \frac{(-1)^{k} q^{k(k+1) / 2} x^{2 k} F^{\prime}(-n+k+1) q^{-n k+n^{2} / 2}}{(q ; q)_{k}} \\
& -x^{-n} \log q \sum_{k=0}^{\infty} \frac{(-1)^{k} q^{k(k+1) / 2} x^{2 k} F(-n+k+1) q^{-n k+n^{2} / 2}(n-k)}{(q ; q)_{k}} .
\end{aligned}
$$

Observe that the fourth and sixth sum can start at $k=n$ since $F(-n+k+1)=0$ if $k<n$. Furthermore, observe that the second and fourth term are equal to

$$
(-1)^{n} q^{n / 2}(q ; q)_{\infty} J_{n}(x ; q) \log x
$$

that the first plus the last term equals

$$
\frac{1}{2} q^{n / 2}(-1)^{n} x^{n} \log q \sum_{k=0}^{\infty} \frac{(-1)^{k} q^{k(k+1) / 2} x^{2 k}(2 k+1) F(k+n+1)}{(q ; q)_{k}}
$$

and that the fifth term equals

$$
\begin{aligned}
& (-1)^{n} q^{n / 2}(q ; q)_{\infty} x^{-n} \log q \sum_{k=0}^{n-1} \frac{(q ; q)_{n-k-1} x^{2 k}}{(q ; q)_{k}} \\
& +(-1)^{n} x^{n} q^{n / 2} \sum_{k=0}^{\infty} \frac{(-1)^{k} q^{k(k+1) / 2} x^{2 k} F^{\prime}(k+1)}{(q ; q)_{n+k}}
\end{aligned}
$$


Hence,

(31)

$$
\begin{aligned}
N_{n}= & 2(-1)^{n} q^{n / 2}(q ; q)_{\infty} J_{n}(x ; q) \log x \\
& +\frac{1}{2} q^{n / 2}(-1)^{n} x^{n} \log q \sum_{k=0}^{\infty} \frac{(-1)^{k} q^{k(k+1) / 2} x^{2 k}(2 k+1) F(k+n+1)}{(q ; q)_{k}} \\
& +(-1)^{n} q^{n / 2}(q ; q)_{\infty} x^{-n} \log q \sum_{k=0}^{n-1} \frac{(q ; q)_{n-k-1} x^{2 k}}{(q ; q)_{k}} \\
& +(-1)^{n} x^{n} q^{n / 2} \sum_{k=0}^{\infty}(-1)^{k} q^{k(k+1) / 2} x^{2 k}\left\{\frac{F^{\prime}(n+k+1)}{(q ; q)_{k}}+\frac{F^{\prime}(k+1)}{(q ; q)_{n+k}}\right\}
\end{aligned}
$$

Substituting (31) and (30) in (29) we obtain

$$
\begin{aligned}
Y_{n}(x ; q)= & \frac{2(q-1)}{\pi \log q} q^{n^{2} / 2} J_{n}(x ; q) \log x-\frac{(1-q)}{\pi} q^{n^{2} / 2} x^{-n} \sum_{k=0}^{n-1} \frac{(q ; q)_{n-k-1} x^{2 k}}{(q ; q)_{k}} \\
& -\frac{(1-q)}{\pi \log q(q ; q)_{\infty}} q^{n^{2} / 2} x^{n} \\
& \times \sum_{k=0}^{\infty}(-1)^{k} q^{k(k+1) / 2} x^{2 k}\left\{\frac{F^{\prime}(n+k+1)}{(q ; q)_{k}}+\frac{F^{\prime}(k+1)}{(q ; q)_{n+k}}\right\} \\
& -\frac{(1-q)}{2 \pi(q ; q)_{\infty}} q^{n^{2} / 2} x^{n} \sum_{k=0}^{\infty} \frac{(-1)^{k} q^{k(k+1) / 2} x^{2 k}(2 k+1) F(n+k+1)}{(q ; q)_{k}} .
\end{aligned}
$$

Now observe that

(33)

$$
\frac{F^{\prime}(n+k+1)}{(q ; q)_{k}}+\frac{F^{\prime}(k+1)}{(q ; q)_{n+k}}=\frac{F(n+k+1)}{(q ; q)_{k}}\left\{\frac{F^{\prime}(n+k+1)}{F(n+k+1)}+\frac{F^{\prime}(k+1)}{F(k+1)}\right\} .
$$

Furthermore, since

$$
F(\nu)=\frac{(q ; q)_{\infty}(1-q)^{1-\nu}}{\Gamma_{q}(\nu)},
$$

we have

$$
\frac{F^{\prime}(\nu)}{F(\nu)}=\frac{-\Gamma_{q}^{\prime}(\nu)}{\Gamma_{q}(\nu)}-\log (1-q) .
$$

With (34) and (33) we can rewrite (32) in a more suitable form. It is

$$
\begin{aligned}
Y_{n}(x ; q)= & \frac{2(q-1)}{\pi \log q} q^{n^{2} / 2} J_{n}(x ; q) \log \frac{x}{1-q} \\
& -\frac{(1-q)}{\pi} q^{n^{2} / 2} x^{-n} \sum_{k=0}^{n-1} \frac{(q ; q)_{n-k-1} x^{2 k}}{(q ; q)_{k}}+\frac{(1-q)}{\pi \log q} q^{n^{2} / 2} x^{n} \\
& \times \sum_{k=0}^{\infty} \frac{(-1)^{k} q^{k(k+1) / 2} x^{2 k}}{(q ; q)_{k}(q ; q)_{n+k}}\left\{\frac{\Gamma_{q}^{\prime}(n+k+1)}{\Gamma_{q}(n+k+1)}+\frac{\Gamma_{q}^{\prime}(k+1)}{\Gamma_{q}(k+1)}\right\} \\
& -\frac{(1-q)}{2 \pi} q^{n^{2} / 2} x^{n} \sum_{k=0}^{\infty} \frac{(-1)^{k} q^{k(k+1) / 2} x^{2 k}(2 k+1)}{(q ; q)_{k}(q ; q)_{n+k}} .
\end{aligned}
$$


Remark. If we replace $x$ by $x(1-q) / 2$ in (35) and take the limit $q \uparrow 1$, we see that the last term disappears and that the remaining three terms become the classical $Y_{n}(x)$ (see [10]).

We will now derive the $q^{1 / 2}$-Wronskian of $J_{\nu}(x ; q)$ and $Y_{\nu}(x ; q)$. With the properties (11) and (12) we find with the $q^{1 / 2}$-Wronskian (20), with $\nu \notin \mathbb{Z}$ (36)

$$
\begin{aligned}
& W_{q^{1 / 2}}\left(J_{\nu}(x ; q), Y_{\nu}(x ; q)\right) \\
& =W_{q^{1 / 2}}\left(J_{\nu}(x ; q), \frac{\Gamma_{q}(\nu) \Gamma_{q}(1-\nu)}{\pi}\left\{\cos (\pi \nu) q^{\nu / 2} J_{\nu}(x ; q)-J_{-\nu}\left(x q^{-\nu / 2} ; q\right)\right\}\right) \\
& =-\frac{\Gamma_{q}(\nu) \Gamma_{q}(1-\nu)}{\pi} W_{q^{1 / 2}}\left(J_{\nu}(x ; q), J_{-\nu}\left(x q^{-\nu / 2} ; q\right)\right) \\
& =\frac{q^{\nu(\nu-1) / 2}\left(1+q^{1 / 2}\right)}{\pi x} .
\end{aligned}
$$

This obviously also holds for $\nu=n \in \mathbb{Z}$.

Note that the $q^{1 / 2}$-Wronskian (36) is never zero for all $\nu$. By Theorem 3 this implies that for all $\nu$ every solution $f$ of (17) can be written as $f(x)=$ $\phi_{1}(x) J_{\nu}(x ; q)+\phi_{2}(x) Y_{\nu}(x ; q)$, where $\phi_{1}(x)$ and $\phi_{2}(x)$ are $q^{1 / 2}$-periodic functions. If we restrict ourselves to solutions of the form $x^{\mu} \sum_{n=0}^{\infty} a_{n} x^{n}$ or $x^{\mu} \log x \sum_{n=0}^{\infty} a_{n} x^{n}$, then every solution of (17) can be written as $c_{1} J_{\nu}(x ; q)+$ $c_{2} Y_{\nu}(x ; q)$, where $c_{1}$ and $c_{2}$ are constants.

\section{REFERENCES}

1. H. Exton, A basic analogue of the Bessel-Clifford equation, Jñānābha 8 (1978), 49-56.

2. __ Basic hypergeometric functions and applications, Ellis Horwood, Chichester, 1983.

3. G. Gasper and M. Rahman, Basic hypergeometric series, Encyclopedia Math. Appl., vol. 35, Cambridge Univ. Press, Cambridge, 1990.

4. W. Hahn, Die mechanische Deutung einer geometrischen Differenzengleichung, Z. Angew. Math. Mech. 33 (1953), 270-272.

5. H. T. Koelink, On quantum groups and q-special functions, $\mathrm{Ph} . \mathrm{D}$. thesis, University of Leiden, 1991.

6. T. H. Koornwinder and R. F. Swarttouw, On q-analogous of the Hankel-and Fouriertransform, Trans. Amer. Math. Soc. 333 (1992), 445-461.

7. L. M. Milne-Thomson, The calculus of finite differences, MacMillan, London, 1951.

8. R. F. Swarttouw, The Hahn-Exton q-Bessel function, $\mathrm{Ph}$. D. Thesis, Delft University of Technology, 1992.

9. L. L. Vaksman and L. I. Korogodskii, An algebra of bounded functions on the quantum group of the motions of the plane, and q-analogues of Bessel functions, Soviet Math. Dokl. 39 (1989), 173-177.

10. G. N. Watson, A treatise on the theory of Bessel functions, second ed., Cambridge Univ. Press, Cambridge, 1966.

Faculteit Wiskunde en Informatica, VRije University, De BoelelaAn $1081 \mathrm{HV}$, AmsTERDAM, THE NETHERLANDS

E-mail address: RENECCS.VU.NL

Faculty of Technical Mathematics and Informatics, Delft University of Technology, Mekelweg 4, 2628CD Delft, The Netherlands 